\title{
Field canalization using anisotropic 2D plasmonics
}

\author{
Po-Han Chang $\mathbb{D}^{1 凶}$, Charles Lin $^{1}$ and Amr S. Helmy ${ }^{1 凶}$
}

Optical devices capable of suppressing diffraction nature of light are of great technological importance to many nanophotonic applications. One important technique to achieve diffractionless optics is to exploit field canalization effect. However, current technological platforms based on metamaterial structures typically suffer from strict loss-confinement trade-off, or lack dynamic reconfigurability over device operations. Here we report an integrated canalization platform that can alleviate this performance trade-off. It is found that by leveraging material absorption of anisotropic 2D materials, the dispersion of this class of materials can flatten without increasing propagation losses and compromising confinement. The realization of such plasmon canalization can be considered using black phosphorus (BP), where topological transition from elliptic to hyperbolic curves can be induced by dynamically leveraging material absorption of BP. At the transition point, BP film can support long range, deeply subwavelength, near-diffractionless field propagation, exhibiting diffraction angle of $5.5^{\circ}$, propagation distance of $10 \lambda_{\mathrm{spp}}$, and $\lambda_{\mathrm{spp}}<\lambda_{0} / 300$.

npj 2D Materials and Applications (2022)6:5; https://doi.org/10.1038/s41699-021-00283-4

\section{INTRODUCTION}

Technological platforms that can suppress the diffraction of light have been studied extensively as they can be utilized in applications that are restricted by diffraction limit such as nearfield spectroscopy, microscopy, energy harvesting, construction of self-trapping devices and free-space optical communications ${ }^{1-5}$. For these applications, the diffraction angle along with propagation loss incurred to alleviate diffraction, are the two key attributes that dictate the performance of such nearly diffractionless optical devices. To date, implementations of low-loss, highly collimated beams typically rely on one of two mechanisms: nonlinear selftrapping $^{6}$, and the propagation of non-diffracting Bessel beams ${ }^{7,8}$. For nonlinear self-trapping, the expansion of optical beams is delicately balanced by nonlinear-induced self-focusing, which collectively permit the beam to propagate without spatial diffraction. Nonetheless, the operation of such spatial soliton requires high input optical powers and is only limited to specific materials endowed with optical nonlinearity. On the other hand, non-diffracting Bessel beams can propagate with no need for nonlinear materials. However, the excitation of Bessel beams requires a delicate control over the phase and amplitude of the wavefronts forming the beams ${ }^{9}$. This inevitably increases the complexity of the system and its operation, particularly in guidedwave settings that are compatible with integrated photonics. Extra structures such as subwavelength nanostructured elements ${ }^{10}$ or photonic crystal structures ${ }^{11,12}$ need to be deployed to assist in the generation of such beams. These resonant structures inherently impact device operational bandwidth and do not offer the ability to dynamically tune the optical properties, making them mostly intended for a limited suite of applications.

An alternative approach that enables the suppression of optical diffraction is to exploit metamaterials and plasmonics ${ }^{4,13,14}$. Through leveraging the material's anisotropy, the dispersion of metamaterials can flatten in the epsilon-near-zero (ENZ) regime, which permits all wave harmonics to propagate in the same direction, allowing the fields to canalize without diffraction. On the other hand, plasmonics can also support optical fields on the subwavelength scale, facilitating the constructions of diffractionless devices in conjunction with achieving such effect with nano-scale confinement ${ }^{5,15}$. This can enhance wave-matter interaction and hence passive and active integrated devices figure of merit. However, the performance of ENZ-based devices is typically compromised by the excessive material absorption that is inherent to the material constituents of such devices, which drastically curtails the propagation length of the canalized beams ${ }^{16}$. In contrast, metamaterials with ultrahigh material absorption are capable of supporting low-loss canalized field ${ }^{17,18}$. In this case, the optical field overlapping lossy materials can be minimized, reminiscent of perfect electric conductor (PEC) effect where the electric field resident in PEC can diminish due to infinite material absorption. Nevertheless, this technique will severely impact optical field confinement, and the high material absorption is also not directly achievable in most of the natural anisotropic materials, hence further implementation of artificial metastructures is required ${ }^{18-20}$. Recently, by stacking two hyperbolic metasurfaces, a highly confined, low-loss optical canalization scheme based on Moire physics has been demonstrated ${ }^{21,22}$. However, the dispersion band depends sensitively on the rotation angle between the two materials, and the operation of canalization regime can only be attained within a narrow range of twisted angle.

In this work, we demonstrate theoretically that with the use of only one layer film anisotropic $2 \mathrm{D}$ material ${ }^{23-25}$, one can achieve a previously unexplored design strategy capable of supporting lowloss canalization of highly confined plasmon waves. By leveraging material absorption which is naturally intrinsic in these materials, it is found that in addition to elliptic and hyperbolic dispersion regimes which are common in ordinary metamaterials, this class of 2D material can possess a combination or a hybrid of elliptic and hyperbolic characteristics. This allows dispersion contours to flatten when transitioning from closed to open dispersion regimes. Notably, such a transition mechanism is not induced by the change in sign of permitivitty in ENZ material or by the control of rotation angle that dictates the hybridization of two metasrufaces, but through the strength of the material absorption. As such, material absorption, which generally represents an

\footnotetext{
${ }^{1}$ The Edward S. Rogers Department of Electrical and Computer Engineering, University of Toronto, Toronto, ON M5S 3G4, Canada. ${ }^{\circledR}$ email: pohan.chang@mail.utoronto.ca; a.helmy@utoronto.ca
} 
unwanted shortcoming for plasmonic structures, can instead be positively exploited to tailor dispersion characteristics of anisotropic 2D material structures and enable new optical functionality. Moreover, the material absorption introduced here will not negatively impact the modal loss and confinement of the plasmonic waves, and the level of material absorption required for canalization is readily available in natural $2 \mathrm{D}$ materials such as black phosphorus (BP) when compared to using PEC effect as shall be demonstrated. The possibilities of dispersion engineering of plasmon modes in 2D anisotropic materials thus render them a promising integrated plasmonic platform for diffractionless optics, while allowing strong tunability over device operations using gate voltage.

\section{RESULTS AND DISCUSSIONS}

\section{Dispersion engineering in anisotropic 2D materials}

Plasmonic waveguides, constructed using 2D plasmonic materials have emerged as a popular optical platform for the realization of photonic devices on a deep subwavelength scale. Plasmonic waveguides using 2D layers can offer the highest level of modal confinement, unprecedented device tunability and enhanced local density of states ${ }^{14,26}$. In particular, 2D plasmonic materials with anisotropic optical properties have received significant attention due to their potential to connect and concomitantly profit from the fields of plasmonics and metamaterials. The versatile dispersion characteristics supported in these 2D structures, enabled by the material's anisotropy, make them an ideal platform for metamaterial-based functions and metacircuit ${ }^{27}$ realized in a 2D flat surface. In this work, we will discuss the opportunities afforded by leveraging material absorption to achieve flatband dispersion within anisotropic 2D materials, which can allow canalization of optical fields in a 2D integrated setting. The dispersion relation of an anisotropic 2D film with in-plane anisotropic conductivity tensor $\sigma_{x, z}$ can be characterized by the following equation ${ }^{28}$ :

$$
\left(k_{x}^{2}-k_{0}^{2}\right) \sigma_{x}+\left(k_{z}^{2}-k_{0}^{2}\right) \sigma_{z}-2 \mathrm{i} \sqrt{k_{x}^{2}+k_{z}^{2}-k_{0}^{2}}\left(\frac{1}{\eta_{0}}+\frac{\eta_{0} \sigma_{x} \sigma_{z}}{4}\right)=0
$$

It is instructive to first consider the effect of material absorption on the dispersion properties in isotropic $2 \mathrm{D}$ materials such as graphene, where the optical properties are characterized by material's in-plane conductivity $(\sigma)$ as schematically shown in Fig. $1 \mathrm{a}$. In the non-retarded regime, the isotropic $2 \mathrm{D}$ dispersion can be obtained by assuming $\sigma_{x}=\sigma_{z}=\sigma$ in Eq. (1), which yields ${ }^{29}$ :

$$
\sqrt{k_{x}^{2} / k_{0}^{2}+k_{z}^{2} / k_{0}^{2}}=\frac{2}{\eta_{0}} \frac{\sigma_{i}+\mathrm{i} \sigma_{r}}{|\sigma|^{2}}
$$

The plots in Fig. 1b, c display the effect of material absorption on isotropic dispersion based on Eq. (2). Without loss of generality, here we assume that the field is propagating in the $z$ direction, and the dispersion behaviors of $k_{z}$ are plotted as function of $\operatorname{Re}\left[k_{x}\right]$, which represents waves of different harmonics. It can be seen that the introduction of material absorption $(\operatorname{Re}[\sigma])$ will lead to the reduction and enhancement in $\operatorname{Re}\left[k_{z}\right]$ and $\operatorname{Im}\left[k_{z}\right]$, respectively, which negatively impacts modal confinement and propagation distance of 2D isotropic plasmons.

In contrast, the mechanism for anisotropic dispersion of 2D materials with material absorption becomes more complex, especially in the regime where $\operatorname{Re}\left[\sigma_{x}\right]>\operatorname{Im}\left[\sigma_{x}\right]$. Initially, we study the dispersion behavior when $\sigma_{x}$ is a real quantity $\left(\operatorname{Im}\left[\sigma_{x}\right]=0\right)$. As detailed in the supplementary material, the dispersion relation in this case can be expressed as:

$$
\sigma_{z} k_{z r}^{4}-\frac{\sigma_{x}^{2}}{4 \sigma_{z}} k_{x}^{4}-\frac{2}{\eta_{0}} \sqrt{\frac{\sigma_{x}}{2 \sigma_{z}}}\left|k_{x}\right| k_{z r}^{2}=0
$$

It can be observed that when $\operatorname{Im}\left[\sigma_{x}\right]=0$, the dispersion of anisotropic $2 \mathrm{D}$ materials can be characterized by a biquadratic equation, which exhibits parabolic features similar to those of hyperbolic dispersion as displayed in Fig. $1 \mathrm{~h}$. It is noteworthy to point out that similar dispersion behavior can also be observed in $3 \mathrm{D}$ metamaterials operated in the ENZ regime $(\operatorname{Im}[\varepsilon]>\operatorname{Re}[\varepsilon] \sim 0)$. In this case, the dispersion can become parabolic even though the real part of permitivity tensor is positive.

The evolution of dispersion of the $\operatorname{Re}\left[k_{z}\right]$ with respect to material absorption $\left(\operatorname{Re}\left[\sigma_{x}\right]\right)$ is further displayed in Fig. 1e-h. It is shown that anisotropic 2D materials can exhibit elliptic, parabolic, and a mixed type of dispersion depending on the value of $\operatorname{Re}\left[\sigma_{x}\right]$. In Fig. 1f, h, the dispersion contours are closed and open curves in the regimes where $\operatorname{Re}\left[\sigma_{x}\right] \ll \operatorname{Im}\left[\sigma_{x}\right]$ and $\operatorname{Re}\left[\sigma_{x}\right] \gg \operatorname{Im}\left[\sigma_{x}\right]$, respectively. On the other hand, the mixed dispersion can occur with moderate material absorption $\left(\operatorname{Re}\left[\sigma_{x}\right] \approx \operatorname{Im}\left[\sigma_{x}\right]\right)$, which exhibits a combination of elliptic and parabolic dispersion as displayed in Fig. 1g.

As evidenced from the results, material absorption offers a path toward dispersion engineering, making it possible to transition from close to open dispersion through the leverage of the strength of material absorption. Such a transition can be verified in Fig. 1i-k, which illustrate the field distributions in 2D anisotropic material launched by a dipole polarized in the out of plane direction. It is shown that a more diffracting, elliptic wavefront is observed in the low material absorption regime, whereas a more confined, hyperbolic-like wavefront can be observed in the high material absorption regime. In the intermediate regime, the anisotropic 2D materials instead can support an elliptic, but a more confined wavefront as depicted in Fig. 1j; a feature corresponding to mixed type of dispersion. It should be highlighted that such a feature cannot be obtained in isotropic materials, where the wavefront is always circular in nature regardless of the strength of material absorption as shown in Fig. $1 \mathrm{~d}$.

It is noteworthy to highlight two important attributes of the mixed type dispersion supported in such anisotropic 2D materials. Because the contour depicts elliptic-like dispersion for smaller $k_{x}$ and parabolic-like dispersion for larger $k_{x}$, there exists a transition point at which the slope $\left(\mathrm{d} k_{z} / \mathrm{d} k_{x}\right)$ of the curve becomes zero. As detailed in the supplementary materials, the value of $k_{x}$ corresponding to this point can be obtained by assuming $\mathrm{d} k_{z} / \mathrm{d} k_{x}=0$ in Eq.(1), which is given by:

$\frac{k_{x}}{k_{0}}=\frac{1}{\eta_{0}\left|\sigma_{x}\right|} \sqrt{\frac{\operatorname{Im}\left[\sigma_{x}\right]}{\operatorname{Im}\left[\sigma_{z}\right]}}$.

On the other hand, in the high $k_{x}$, non-retarded regime, the slope of dispersion curve in the parabolic/hyperbolic-like regime is also derived in the supplementary material, and can be expressed as:

$\frac{\mathrm{d} \operatorname{Re}\left[k_{z}\right]}{\mathrm{d} k_{x}}=\sqrt{\frac{\operatorname{Re}\left[\sigma_{x}\right]}{2 \operatorname{lm}\left[\sigma_{z}\right]}}$.

From Eqs. 4 and 5, it is seen that in this case the slope of the curve in the high $k_{x}$ regime is predominantly characterized by material absorption, which in turn can confirm the dispersion curve obtained in the high $\operatorname{Re}\left[\sigma_{x}\right]$ regime in Fig. $1 \mathrm{~h}$.

In the field of metamaterials, similar mixed/hybrid dispersion can also occur in bianisotropic materials ${ }^{30}$, which are described by high order dispersion relations with circularly or elliptically polarized eigenwaves, or parity time (PT) symmetry 
(b)

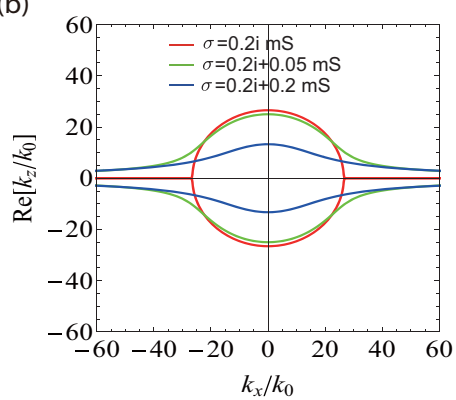

(a)

(c)

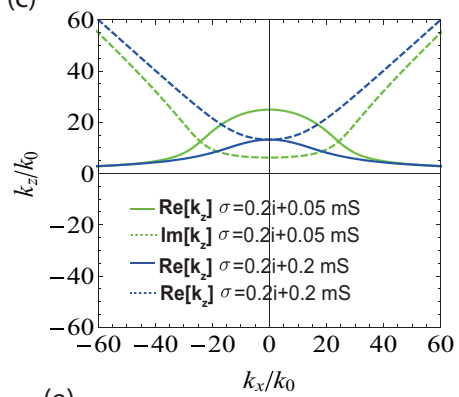

(d)

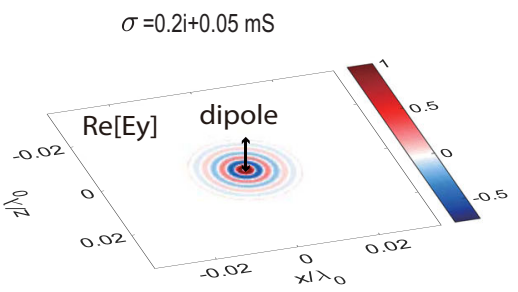

(e)

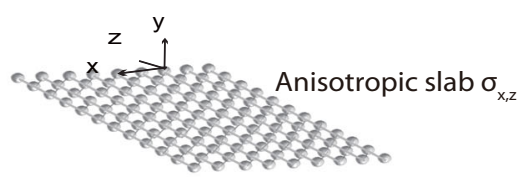

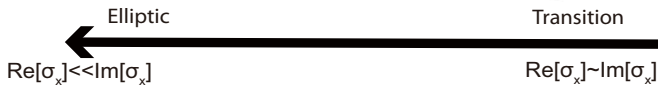

(f)

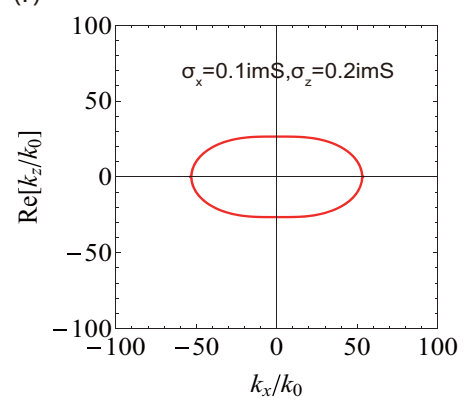

(i)

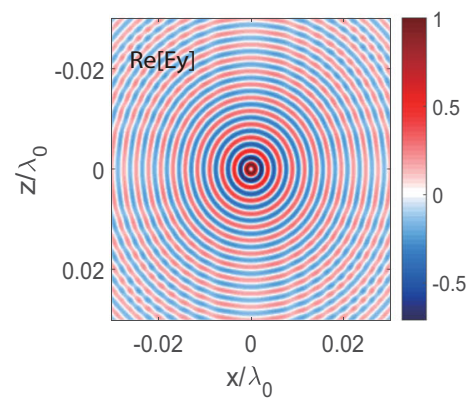

(g)

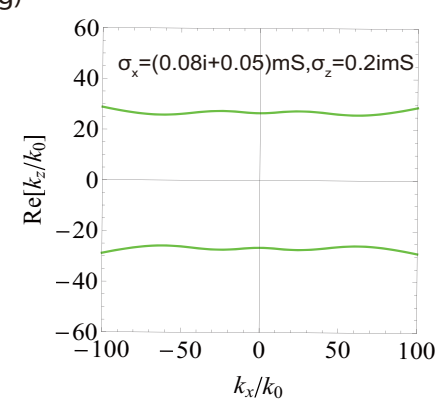

(j)

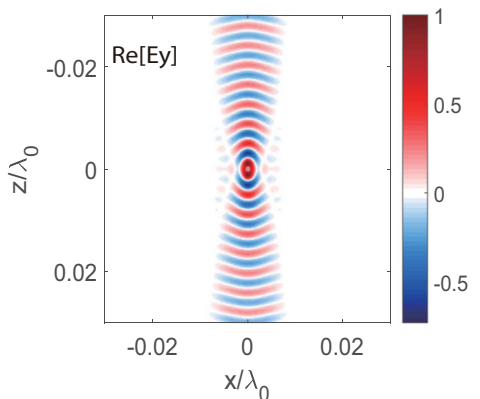

Hyperbolic-like

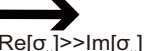

(h)

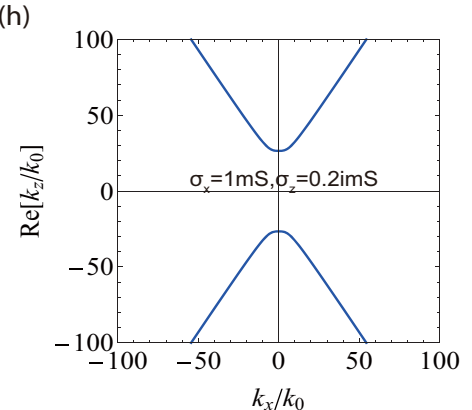

(k)

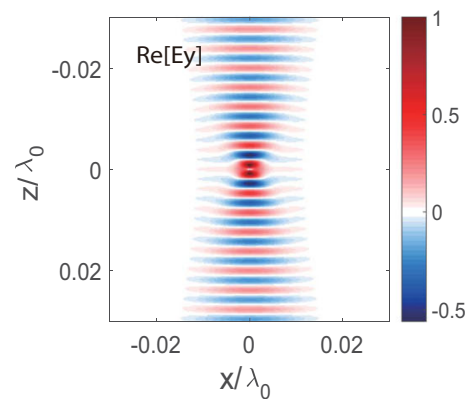

Fig. 1 Dispersion of anisotropic 2D materials. a Schematic of an isotropic 2D material sitting on the $x-z$ plane, which is optically characterized by in-plane conductivity $\sigma$. Dispersion contours of $(\mathbf{b}) \operatorname{Re}\left[k_{z}\right]$ and $(\mathbf{c}) \operatorname{Im}\left[k_{z}\right]$ of an isotropic $2 \mathrm{D}$ material as function of $k_{x}$ using various values of $\operatorname{Re}[\sigma]$. It is seen that the increase in material absorption $(\operatorname{Re}[\sigma]$.$) will lead to the reduction in \operatorname{Re}\left[k_{z}\right]$ and the increase in $I m\left[k_{z}\right]$, which is considered as a shortcoming for 2D plasmon due to reduced propagation distance. d For isotropic 2D films, the wavefront launched by a $y$-polarized electric dipole is always circular regardless of the strength of material absorption. e Schematic of an anisotropic 2D material characterized by an in-plane anisotropic conductivity tensor $\left(\sigma_{x, z}\right)$. Contrary to isotropic 2D films, anisotropic 2D materials can support (f) elliptic, (h) hyperbolic, and (g) mixed type of dispersion depending on the strength of material absorption $\left(\operatorname{Re}\left[\sigma_{x}\right] / \operatorname{Im}\left[\sigma_{x}\right]\right)$. For mixed dispersion, the dispersion contour can possess a hybrid character of the elliptic and hyperbolic dispersions. When launched by a $y$-polarized dipole, anisotropic 2D films therefore can support (i) elliptic, or (k) hyperbolic field distributions in the low and high material absorption regimes. (j). In the intermediate regime, mixed type of field distribution can be supported, which offers a pathway toward dispersion transition.

metamaterials ${ }^{31}$ which involve spatial modulation of loss and gain. However, these approaches involve the interaction of light with subwavelength nanoparticles and are mostly intended for freespace applications due to the flow of light from and into surfaces orthogonally, rather than in-plane. In contrast, naturally occurring 2D materials are better suited as an integrated in-plane platform due to their structural simplicity. The mixed type of dispersion afforded in anisotropic 2D materials therefore can facilitate new integrated optical functionalities that are not possible in conventional elliptic and hyperbolic metamaterials. Markedly, it indicates the possibility of topological transition from elliptic to hyperbolic dispersion as a functional regime where devices can be built and 
(a)

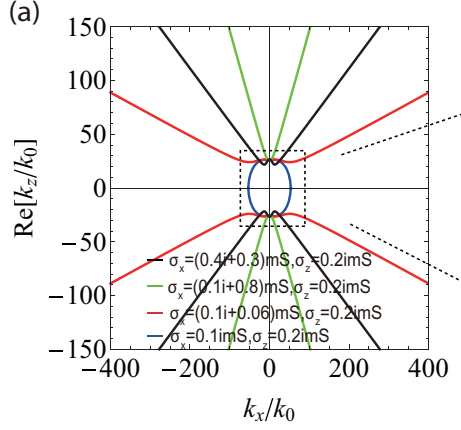

(d)

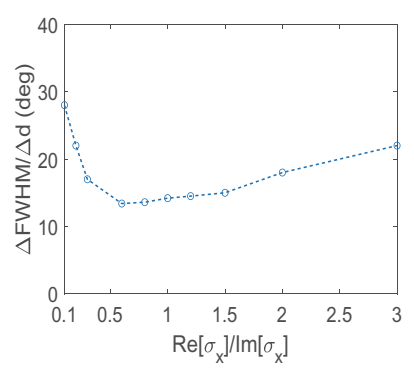

(b)

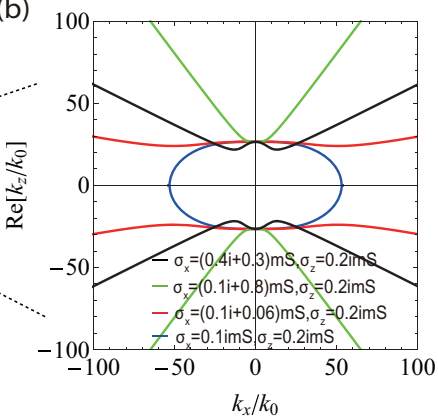

(e)

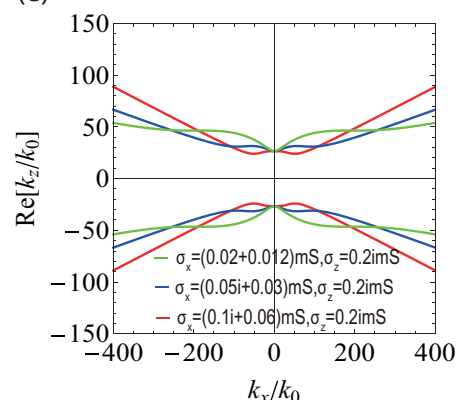

(c)

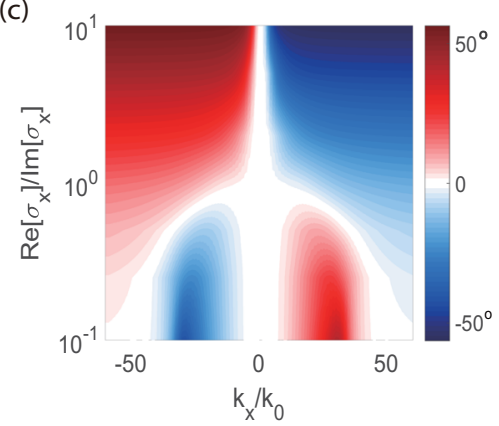

(f)

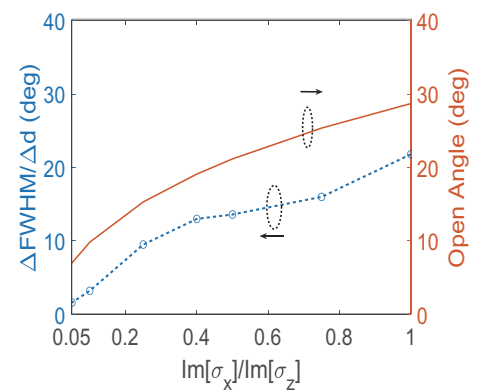

Fig. 2 Flatband dispersion in anisotropic 2D materials. a Dispersion curves of anisotropic 2D materials using various combinations of Re $\left[\sigma_{x}\right]$, and $\operatorname{Im}\left[\sigma_{x}\right]$, which can support elliptic, hyperbolic and mixed type of dispersion by regulating material absorption, allowing contour to transition from close to open. b Detailed view of the dispersion curves shown in (a). It is seen that when $\operatorname{Re}\left[\sigma_{x}\right] \sim \operatorname{Im}\left[\sigma_{x}\right]$, the dispersion curve can be flattened in the regime where $-100<k_{x} / k_{0}<100$ as a result of mixed type of dispersion. $c$ The angle of the direction of Poynting vector $\left(\partial \mathrm{k}_{\mathrm{z}} / \partial \mathrm{k}_{\mathrm{x}}\right)$ of $\mathrm{k}_{\mathrm{x}}$ modes as a function of material absorption with respect to $z$-axis. When $\operatorname{Re}\left[\sigma_{x}\right] \sim \operatorname{Im}\left[\sigma_{x}\right]$, the angle of Poynting vector can become zero over a broad range of $k_{x}$, permitting canalization to occur. d Numerically simulated diffraction angle ${ }^{21}$ of the field propagation in anisotropic $2 \mathrm{D}$ materials $\left(\operatorname{Im}\left[\sigma_{x, z}\right]=0.1,0.2 \mathrm{imS}\right)$ as function of material absorption $\left(\operatorname{Re}\left[\sigma_{x}\right] / \operatorname{Im}\left[\sigma_{x}\right]\right)$ launched by a dipole source. The dipole source is pointing in the out-of-plane $(y)$ direction and will excite all $k_{x}$ spatial harmonics. It is seen that the diffraction can be best suppressed when $\operatorname{Re}\left[\sigma_{x}\right] \approx 0.6 \mathrm{Im}\left[\sigma_{x}\right]$. e Dispersion curves in anisotropic $2 \mathrm{D}$ materials with varying degree of in-plane anisotropy $\left(\operatorname{Im}\left[\sigma_{z}\right] / \operatorname{Im}\left[\sigma_{x}\right]\right)$ while using the same strength of material absorption $\left(\operatorname{Re}\left[\sigma_{x}\right]=0.6 \operatorname{lm}\left[\sigma_{x}\right]\right)$. It is shown that on top of material absorption, it is possible to further flatten dispersion curve with the aide of more extreme material's anisotropy. ( $f$ ) Diffraction and open angels of the propagating fields in anisotropic $2 \mathrm{D}$ materials $\left(\operatorname{Im}\left[\sigma_{x}\right]=0.2 \mathrm{mS}\right.$ and $\left.\left.\operatorname{Re}\left[\sigma_{x}\right]=0.6 \mathrm{Im}\left[\sigma_{x}\right]\right)\right)$ as a function of in-plane anisotropy launched by a y-directed dipole source. Diffraction can be further suppressed by decreasing the value of $\operatorname{Im}\left[\sigma_{x}\right] / \operatorname{Im}\left[\sigma_{z}\right]$ as a result of more flatted dispersion enabled by anisotropy.

operated. Such a transition is of great interest to plasmonic metadevices because the dispersion contour can be tailored by regulating material absorption. In the next section, we will demonstrate how to further leverage such dispersion characteristics to achieve plasmon canalization in 2D materials for diffractionless optics.

\section{Field canalization in anisotropic 2D materials facilitated by material absorption}

The mixed type dispersion in anisotropic 2D materials can provide new opportunities to achieve optical functions unattainable in their isotropic counterparts. In particular, the existence of a transition point along with the control over the slope of the curve in the open regime, allow the dispersion to flatten by leveraging material absorption. Because the slope in the elliptic and hyperbolic regimes are predominantly dictated by degree of anisotropy $\left(\operatorname{Im}\left[\sigma_{x}\right] / \operatorname{Im}\left[\sigma_{z}\right]\right)$ and the material absorption respectively (Eq. 5), one can define the following condition to achieve flatband dispersion in anisotropic 2D materials:

$\operatorname{Im}\left[\sigma_{z}\right]>\operatorname{Im}\left[\sigma_{x}\right] \approx \operatorname{Re}\left[\sigma_{x}\right]$

Figure $2 \mathrm{a}$, $\mathrm{b}$ illustrate an example as to how to leverage material absorption to achieve flatband dispersion based on Eq.(6). It is seen that for an anisotropic 2D film free of material absorption $\left(\operatorname{Re}\left[\sigma_{x}\right]=0\right)$, the dispersion curve will exhibit an elliptic characteristics when $\operatorname{Im}\left[\sigma_{x, z}\right]=0.1,0.2 \mathrm{imS}$ as depicted by the blue curve in Fig. 2a. However, by increasing the strength of material absorption such that $\operatorname{Re}\left[\sigma_{x}\right] \approx \operatorname{Im}\left[\sigma_{x}\right]$, the initial, closed, elliptic dispersion contour can become open and the mixed type of dispersion will be supported. The opening angle of the curve can be tuned by using various values of $\operatorname{Re}\left[\sigma_{x}\right] / \operatorname{Im}\left[\sigma_{z}\right]$ as depicted by the red $\left(\sigma_{x}=\right.$ $0.1 \mathrm{i}+0.06 \mathrm{mS})$ and black $\left(\sigma_{x}=0.4 \mathrm{i}+0.3 \mathrm{mS}\right)$ curves. On the other hand, the dispersion curve will become predominantly hyperbolic when $\operatorname{Re}\left[\sigma_{x}\right]$ further increases, losing the elliptic feature as plotted by the green curve in Fig. 2a.

Such a material absorption-induced transition can be further examined in Fig. $2 b$, which details the dispersion characteristics in the regime wherein $-100<k_{x} / k_{0}<100$. Due to the emergence of a transition point pertaining to the mixed type of dispersion, a more flattened contour curve can be obtained in this regime when $\operatorname{Im}\left[\sigma_{x}\right]<\operatorname{Im}\left[\sigma_{z}\right]$ with the use of moderate material absorption as given by Eq.(5). This dispersion feature, in turn, can facilitate field canalization in anisotropic 2D materials, which requires the Poynting vector of different $k_{x}$ modes to have the same direction. As depicted in Fig. 2c, the Poynting vector can become unidirectional $(\theta=0)$ for a broad range of $k_{x}$ modes when $\operatorname{Re}\left[\sigma_{x}\right] \approx \operatorname{Im}\left[\sigma_{x}\right]$, hence allowing modes of different spatial harmonics to canalize in the same propagation direction.

When launched by a dipole source, the canalization effect enabled by flattened dispersion can suppress the diffraction of the propagating field, permitting a broad range of $k_{x}$ harmonics to propagate in the same direction. To assess the effect of canalization induced by material absorption, we compare the canalization angle, defined by $\Delta \mathrm{FWHM} / \Delta \mathrm{d}^{22}$, as function of $\operatorname{Re}\left[\sigma_{x}\right]$ as plotted in Fig. $2 \mathrm{~d}$, where FWHM is the full width half maximum of the wavefront and $\Delta \mathrm{d}$ is the propagation distance of the fields. The expansion of the wavefront can be optimally suppressed 
when $\operatorname{Re}\left[\sigma_{x}\right] \approx 0.6 \operatorname{Im}\left[\sigma_{x}\right]$. In this case, the canalization angle can be as small as $13^{\circ}$, indicative of the possibility of field canalization through the regulation of material absorption.

It is important to note that diffraction can be further suppressed through the interplay between material absorption and anisotropy for the best performance of canalization. As displayed in Fig. $2 \mathrm{f}$, it is seen that with the same level of material absorption $\left(\operatorname{Re}\left[\sigma_{x}\right]=0.6 \operatorname{Im}\left[\sigma_{x}\right]\right)$ being used, the canalization angle can be reduced further by increasing the degree of material anisotropy $\left(\operatorname{Im}\left[\sigma_{z}\right] / \operatorname{Im}\left[\sigma_{x}\right]\right)$. This is due to a broader range of canalized $k_{x}$ modes achievable in anisotropic $2 \mathrm{D}$ materials when using a stronger degree of material's anisotropy on top of material absorption, as illustrated in Fig. 2e. Also shown in the plot is the open angle, which can offer an analytical estimation of the canalization effect based on the slope in the hyperbolic regime $\left(\tan ^{-1}\left(\operatorname{Re}\left[\sigma_{x}\right] / 2 \operatorname{lm}\left[\sigma_{z}\right]\right)\right)$. It is evident that the trend in the canalization angle shows good agreement with that of open angle, which suggests that the stronger canalization effect is because dispersion can be flattened into the higher $k_{x}$ regime as shown by the green curve in Fig. 2e.

\section{Loss and confinement trade-off in 2D canalized structures}

In this section, we will show how the material absorption-induced flatband dispersion can be engineered to enable low loss, highly confined canalized fields when compared to other canalized techniques based on ENZ and PEC effects. This can address the design bottleneck that is common in plasmonic devices, where material absorption typically dictates strict trade-offs between modal loss and confinement. For instance, for canalization through using ENZ effects, the material absorption will greatly reduce the propagation length of the canalized plasmon fields whereas in the PEC scheme, low-loss canalized plasmon fields can be obtained but subwavelength confinement of the modes will be compromised. It should be noted that similar design trade-off has been recently addressed using composite hybrid plasmonic structures, which can facilitate device performance of various plasmonic functional devices ${ }^{32,33}$. Anisotropic 2D materials can instead offer a platform to achieve superior canalized mode attributes without the need for implementing layered waveguide structures, while simultaneously offering strong device tunability.

Counter-intuitively, the low-loss canalized field achieved in this work is facilitated by regulating material absorption such that $\operatorname{Re}\left[\sigma_{x}\right] \approx \operatorname{Im}\left[\sigma_{x}\right]$. This is in stark contrast to conventional design wisdom, which dictates that the formation of low-loss plasmonic modes should require the functional plasmonic materials be operated either in low or ultrahigh material absorption regimes, such as the ones in ENZ and PEC canalized schemes respectively. To understand how moderate material absorption can impart such a low-loss canalization effect, in the supplementary material we study the dispersion behavior of the real and imaginary components of $k_{z}$ as a function of $k_{x}$. As can be seen, the material absorption introduced here has a disproportional effect on the modal loss in the elliptic and parabolic regimes, as $\operatorname{Im}\left[k_{z}\right]$ increases more significantly only after the transition point into the hyperbolic regime. As such, a broad range of low-loss $k_{x}$ modes can still be achieved in the elliptic regime, which in turn permits low-loss canalized field propagation.

An important figure of merit for plasmonic modes is the ratio of $\operatorname{Im}\left[k_{z}\right]$ over $\operatorname{Re}\left[k_{z}\right]^{15,34}$, which reflects the length of propagation distance of the plasmon modes normalized to the wavelength of operation. Additionally, confinement of the modes can be estimated by the penetration depth $(L)$ into the vacuum, as defined by $\operatorname{Im}\left[k_{y}\right] / 2 \lambda_{0}$. Such a metric has been utilized widely to characterize the confinement of plasmonic modes. Based on these two metrics, we can compare the modal loss and confinement of different 2D canalized schemes as functions of material absorption and $k_{x}$, which are plotted in Fig. 3.
As displayed in Fig. 3a-c, the increased material absorption in the scheme we describe in this work can enable a relatively broadband, low-loss dispersion in the elliptic operating regime. Furthermore, the modal confinement is nearly unaffected, indicating that all spatial harmonics can still carry deeply subwavelength information, despite the presence of material absorption.

Conversely, the material absorption in ENZ canalized scheme represents an undesired shortcoming to the quality of plasmon modes. As can be seen in Fig. 3d-f, the increased material absorption will negatively impact the modal loss and confinement, therefore degrading the performance of canalized plasmon fields. As such, material absorption should be minimized for the best performance of ENZ canalization. On the other hand, in the PEC scenario shown in Fig. $3 g-i$, the $2 D$ materials should be operated in the ultrahigh regime to support low-loss plasmon canalized fields. However, as displayed in Fig. $3 \mathrm{i}$, the modal confinement will be severely compromised in the low $k_{x}$ regime, inevitably losing subwavelength features originally offered by $2 \mathrm{D}$ plasmoncis. Similar design trade-off associated with ENZ and PEC canalized schemes can also be observed in their 3D metamaterial version as discussed in the supplementary material. Overall, as showcased in Fig. 3a, $d$ and $g$, the afforded mixed type of dispersion can better alleviate the associated loss-confinement trade-off by moderately regulating material absorption, offering a new avenue for the construction of diffractionless devices with improved performance. Compared with other canalized platforms, the material absorption inherent in anisotropic 2D materials can therefore favor low-loss plasmon canalization in the deep subwavelength regime.

\section{Anisotropic 2D materials as a gate-tunable canalization platform}

In this section, we will demonstrate how to leverage material absorption of natural 2D anisotropic materials such as BP to realize low loss, highly confined canalized modes. Recently, BP plasmonics operating in the mid-infrared (MIR) regime have received significant interest for nanophotonic applications such as sensing, molecular analysis, and optical communications ${ }^{23,35}$. Here we shall showcase that it can also serve as a reconfigurable, low-loss canalized platform in these important wavelength regimes. One prominent advantageous feature of anisotropic $2 \mathrm{D}$ material over bulk metamaterials is the ability to tune the conductivity tensor by chemical potential or electric bias, which can offer strong tunability over wavefront manipulation in BP film as schematically depicted in Fig. 4a. For instance, Fig. 4b displays the conductivity tensor of $20 \mathrm{~nm}$ BP as a function of chemical potential in the MIR regime when $\lambda_{0}=3.25 \mu \mathrm{m}$, where $x$ and $z$ are the armchair and zigzag directions of $\mathrm{BP}$, respectively. The strength of material absorption in $\sigma_{x}$ can be tuned significantly as a function of applied chemical potential. The canalization condition based on Eq.(6) can be therefore achieved in the vicinity of $\mu=0.08 \mathrm{eV}$, where $\operatorname{Re}\left[\sigma_{x}\right] \approx \operatorname{Im}\left[\sigma_{x}\right]$.

A dynamical topological transition from elliptic $(\mu=0.1 \mathrm{eV})$ to hyperbolic $(\mu=0.06 \mathrm{eV})$ contours can be realized, while the transition point corresponds to flattened dispersion $(\mu=0.08 \mathrm{eV})$ as shown in Fig. 4c. BP film operated with this condition can enable highly collimated canalized fields as illustrated in the field distributions within Fig. 4e. In this case, BP can support long range, highly canalized field with a diffraction angle of $5.5^{\circ}$ and propagation distance of $5 \lambda_{\text {spp }}$, highlighting $B P^{\prime} s$ potential as a tunable diffractionless material platform. It should be noted that the transition mechanism here is induced by regulating the material absorption, provided that the dispersion of the functional anisotropic materials can sweep across the point where the strength of material absorption is moderate $\left(\operatorname{Re}\left[\sigma_{x}\right] \approx \operatorname{Im}\left[\sigma_{x}\right]\right)$. On the other hand, the diffraction angle of BP operated under the 
(a)
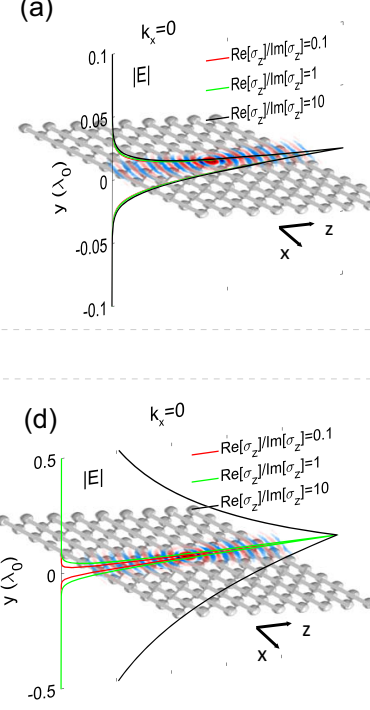

(g)

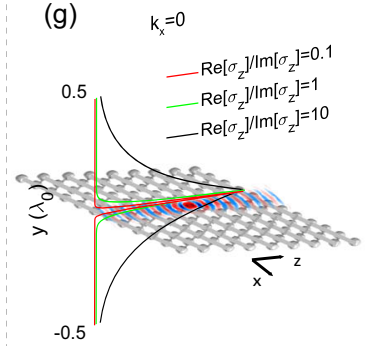

(b) Modal loss

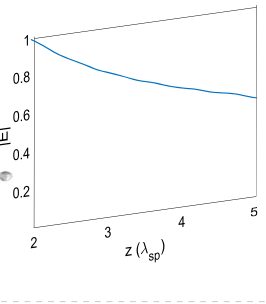

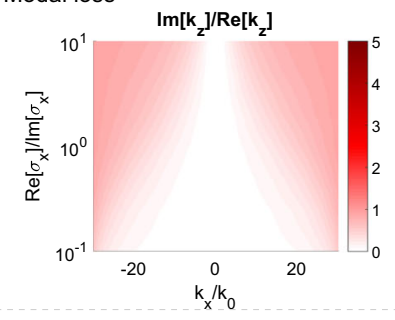

ENZ canalization $\sigma_{z}=0.2 \mathrm{i}+\operatorname{Re}\left[\sigma_{z}\right] \mathrm{mS}, \sigma_{\mathrm{x}}=0.01 \mathrm{imS}$

(e) Modal loss

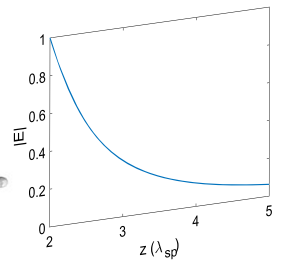

(c) Modal confinement

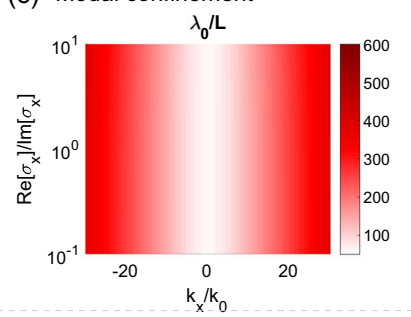

(f) Modal confinement

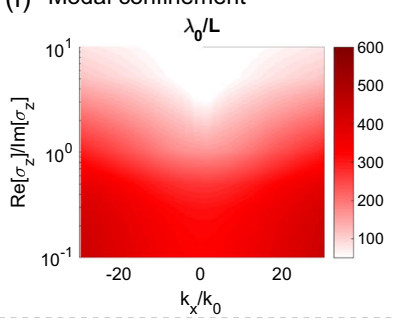

PEC canalization $\sigma_{x}=0.1 \mathrm{imS}, \sigma_{-}=0.2 \mathrm{i}+\operatorname{Re}\left[\sigma_{z}\right] \mathrm{mS}$

(h) Modal loss

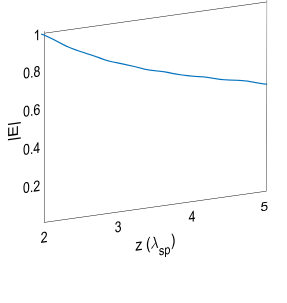

(i) Modal confinement

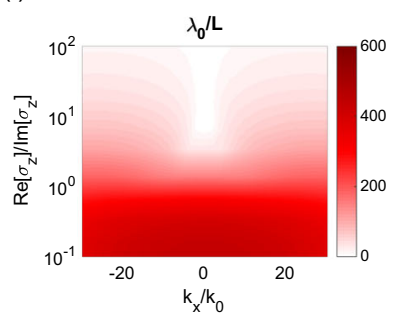

Fig. 3 Loss-confinement for different canalized schemes. Comparison of modal loss $\left(\operatorname{Im}\left[k_{z}\right] / \operatorname{Re}\left[k_{z}\right]\right)$ and confinement $\left(L / \lambda_{0}\right)$ in different $2 D$ canalized platforms based on: (a)-(c) material absorption shown in this work (d)-(f) ENZ effect, and (g)-(i) PEC effect. Larger penetration depth $(L)$ will lead to weaker modal confinement. a Schematic, penetration depth as function of material absorption $\left(k_{x}=0\right)$, and the power attenuation $\left(\sigma_{x, z}=0.1 \mathrm{i}+0.06,0.2 \mathrm{imS}\right)$ of the canalized field in anisotropic $2 \mathrm{D}$ materials facilitated by material absorption. $\mathbf{b}$ Modal loss and (c) modal confinement of anisotropic 2D modes as functions of $k_{x} / k_{0}$ and material absorption. It is seen that a broad range of low-loss $k_{x}$ modes can still be supported even with the introduction of material absorption, while the modal confinement remains largely non-impacted as shown in (a). d Schematic, penetration depth, and the power attenuation of the canalized field facilitated by ENZ metasurface $\left(\sigma_{x, z}=0.2 i+\right.$ $0.06,0.001 \mathrm{imS})$, where it is seen that the propagation distance will be greatly curtailed with the introduction of material absorption. e Modal loss and (f) modal confinement of ENZ metasurface as functions of $k_{x} / k_{0}$ and material absorption. The performance of modal loss and modal confinement will be degraded with the increase of material absorption, particularly in small $k_{x}$ regime. g Schematic, penetration depth, and the power attenuation of the canalized field facilitated by PEC effect. It is seen that the modes operated in PEC condition will become loosely guided, losing subwavelength feature offered by 2D plasmonics. $\mathbf{h}$ Modal loss and (i) modal confinement of PEC metasurface as functions of $k_{x} / k_{0}$ and material absorption. As can be seen, the modal loss can be reduced however the modal confinement will be degraded in the PEC regime.

ENZ condition $(\mu=0.075 \mathrm{eV})$ will increase to $15^{\circ}$ with a reduced propagation distance of $2 \lambda_{\text {spp. }}$. In this case, the dispersion curve will become hyperbolic-like which will lead to a more diffracting field propagation. Since the strength of material absorption can be tuned through chemical potential or bias in $2 \mathrm{D}$ materials, such a transition can be viable in most of the anisotropic $2 D$ materials but not limited to BP.

Table 1 further compares the optical performance of various canalization platforms, where metamaterials can be realized using metal-dielectric structures ${ }^{19,20}$. There are at least two major dispersion characteristics that are distinct from those of BP counterpart. First, the range of $k_{x}$ modes in the canalization regime in metamaterials $\left(-5<k_{x} / k_{x}<5\right)$ is about two order magnitude smaller than that of BP $\left(-300<k_{x} / k_{x}<300\right)$, which hinders higher $k_{x}$ harmonics from contributing canalization process. As such, BP plasmonics allow for better neardiffractionless propagation as compared with metamaterials, with diffraction angle of only $5^{\circ}$. Second, the value of $\operatorname{Re}\left[K_{z}\right]$ in the canalized regime in metamaterials based on metal is also around two order magnitude smaller than that of BP. The capability of 2D materials such as BP to support highly confined surface mode with guided wavelength $\left(\lambda_{\text {spp }}\right)$ much smaller than $\lambda_{0}$ make them a more favorable platform of choice for one to realize canalized fields in strongly subwavelength regime. In contrast, the guided wavelength supported in metamaterial or metal plasmonics will be within the same order as $\lambda_{0}$. In order to, account such subwavelength feature enabled by $2 \mathrm{D}$ materials, the figure of merit for the modal loss of $2 \mathrm{D}$ plasmonic is defined by $\operatorname{Im}\left[k_{z}\right] / \operatorname{Re}\left[k_{z}\right]$, which indicates the propagation distance normalized to guided wavelength ${ }^{15}$. Clearly, through the regulation of material absorption, anisotropic 2D plasmonic material can represent a new paradigm for the constructions of diffractionless devices, while simultaneously facilitating long range, highly canalized field in the deep subwavelength regime. 
(a)

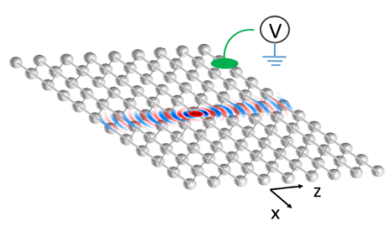

(b)
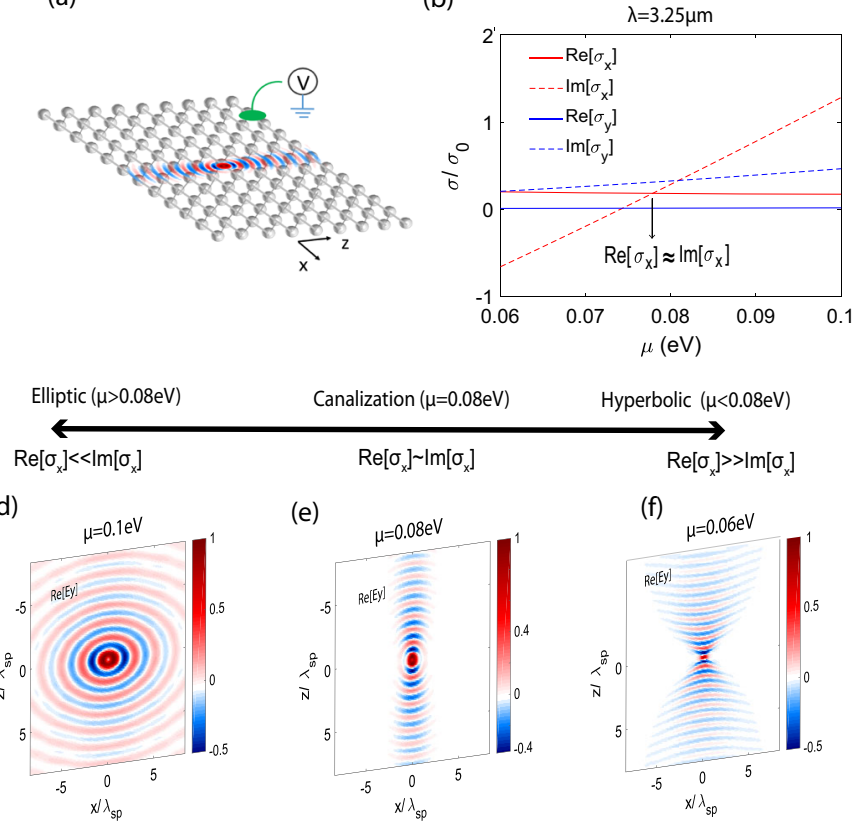

Canalization $(\mu=0.08 \mathrm{eV})$

$\operatorname{Re}\left[\sigma_{x}\right] \sim \operatorname{Im}\left[\sigma_{x}\right]$

(e)

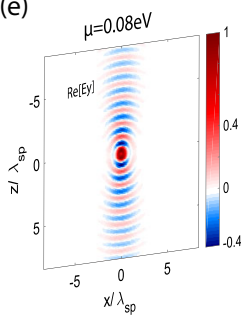

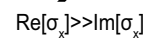

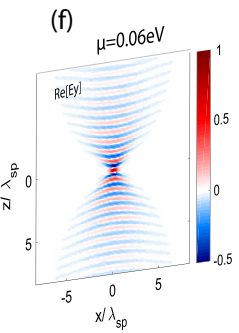

Hyperbolic $(\mu<0.08 \mathrm{eV})$ (c)

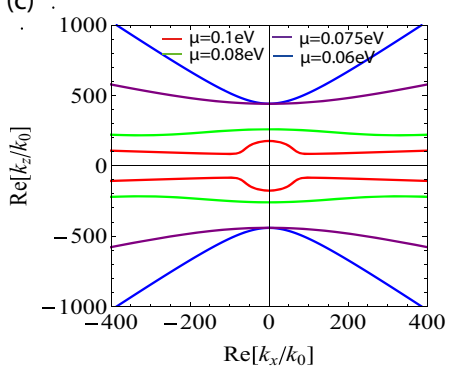

(g)

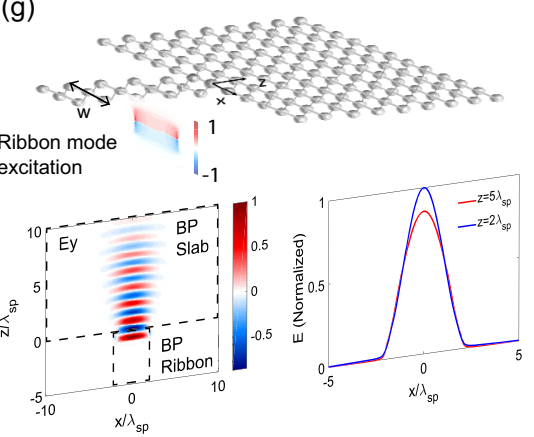

Fig. 4 BP as a gate-tunable 2D canalization platform. a Schematic of BP film being considered for the realization of low-loss canalization field tunable by bias or chemical potential. b Normalized conductivity tensor $\left(\sigma_{0}=e^{2} / 4 \hbar\right)$ in the armchair $(x)$ and zigzag $(z)$ directions for $20 \mathrm{~nm} \mathrm{BP}$ as a function of chemical potential ${ }^{23,24}$ when $\lambda_{0}=3.25 \mu \mathrm{m}$. The conductivity of BP can be highly tunable where various material absorption level can be achieved via bias or chemical doping. In this case, moderate material absorption can be attained in BP when $\mu=$ $0.08 \mathrm{eV}$. c Material absorption-facilitated topological transition in BP when $\lambda_{0}=3.25 \mu \mathrm{m}$. The dispersion contour can be elliptic, flattened, and hyperbolic when, respectively, showing strong tunability over dispersion characteristics. The dispersion of BP under ENZ condition $(\mu=0.075 \mathrm{eV})$ is also plotted for comparison. d-f Field distributions in such functional BP film using different chemical potential, allowing dynamical control over the dispersion and wavefronts manipulation with different diffraction characteristics. When $\mu=0.08 \mathrm{eV}$, the diffraction angle is calculated to be only $5.5^{\circ}$, which can support near-diffractionless propagation of plasmon field. $\mathbf{g}$ Demonstration of an in-plane, integrated canalization platform using BP $(\mu=0.08 \mathrm{eV})$ when excited by a ribbon mode of finite width, where the Gaussian field profile of the ribbon mode supported in BP ribbon waveguide $(z<0)$ can be maintained even in the regime when the width of BP becomes unbounded $(z>0)$, exhibiting diffractionless feature in an in-plane setting.

Table 1. Performance comparison of various canalization platforms.

\begin{tabular}{|c|c|c|c|c|c|}
\hline Canalized scheme & Platform & Diffraction angle & Propagation length & Guided wavelength $\left(\lambda_{\text {spp }}\right)$ & Penetration depth \\
\hline Metamaterial ENZ & Free space & $10^{\circ}$ & $0.25 \lambda_{\mathrm{spp}}$ & $\approx \lambda_{0} / 1.2$ & $\approx \lambda_{0} / 15$ \\
\hline Bilayer metasurfaces ${ }^{22}$ & Free space & $6.6^{\circ}$ & $5 \lambda_{\mathrm{spp}}$ & $\approx \lambda_{0} / 50$ & $\approx \lambda_{0} / 500$ \\
\hline BP (Material absorption) & Integrated & $5.5^{\circ}$ & $5 \lambda_{\text {spp }}$ & $\approx \lambda_{0} / 300$ & $\approx \lambda_{0} / 4000$ \\
\hline
\end{tabular}

The construction of metamaterial based on PEC effect is realized using layered structures involving zirconium nitride (ZrN) and a dielectric with $\epsilon=10^{20}$, while the metamaterial based on ENZ effect can be considered involving AZO and TiO2. PEC scheme based on BP can also be considered with the use of periodic BP nanoribbons ${ }^{18}$. Unlike BP plasmonics, these platforms are unable to support modes in the deep subwavelength regime. Penetration depth of the modes $\left(k_{x}=0\right)$ is also shown.

\section{In-plane, integrated canalization platform in 2D material structures}

In this section, we will discuss the attributes of anisotropic 2D materials as an attractive integrated photonic platform where field canalization can be configured in an in-plane, guided-wave setting without the need for combining other cladding layers to create 3D stacks. Integrated photonic devices can offer greater stability, density and scalability than their free space counterpart. Most importantly, by exploiting the anisotropic optical properties, these anisotropic materials can play the role of optical nanocircuit elements analogous to microelectronics ${ }^{27}$. Thus, an integrated platform that can support low loss, subwavelength field canalization with dynamic reconfigurability is highly desired. However, these important attributes may not be obtainable simultaneously with the use of current technological platforms.

For instance, with the use of twisted bilayer hyperbolic metasurfaces such as $\mathrm{a}-\mathrm{MoO}_{3}$, a tunable, low loss, deeply subwavelength, and canalized plasmon scheme can be achieved $^{21}$. Such a platform is based on the dispersion hybridization between anisotropic plasmon polartion fields in stacked a$\mathrm{MoO}_{3}$, reminiscent of the peculiar electronic band structure modified by van-der-Waals superlattices ${ }^{36}$. More recently, it is demonstrated that such a bilayer twisted structure can be deployed to effectively create longitudinal spin of plasmon necessary for chiral plasmonics ${ }^{37,38}$. However, despite the numerous emerging photonic applications that twisted optics 
can enable, the requirement of stacking two films in the out-ofplane direction makes it more suitable for free space optical elements. Additionally, the dispersion of such bilayer structures depends sensitively on the twisted angle between the films, which is not tunable therefore limits its capabilities for active applications. On the other hand, a coplanar scheme based on the PEC ${ }^{4}$ effect has also been proposed to achieve plasmon canalization. However, the operation of this scheme should require the implementation of composite subwavelength metastructures to achieve the desired material parameters. The actual canalization performance, based on such periodic structures, will be restricted by optical nonlocality ${ }^{25}$, particularly in the in-plane, integrated setting where such nonlocal optical effect will be more pronounced with the presence of in-plane wave vector. Additionally, similar to bilayer hyperbolic canalized scheme, the implementations of densely packed multilayer structures in these platforms will lead to higher optical loss more stringent to material granularity and impurity, which degrades the quality of canalized plasmon fields. In contrast, the canalization scheme facilitated by material absorption proposed in this work will not have these challenges as flatband engineering can be accomplished with the use of only single anisotropic 2D film which can be gate tunable in an in-plane, integrated setting.

To highlight the possibility of anisotropic 2D material as an integrated canalization platform, an in-plane excitation scheme involving an unbounded BP film connected to a BP ribbon waveguide of finite width can be considered, as schematically depicted in Fig. 4g. BP can suppress optical diffraction even when the ribbon mode $(z<0)$ enters the unbounded regime $(z>0)$, maintaining highly confined Gaussian field profiles as the mode propagates down further into the film when $\mu=0.08 \mathrm{eV}$. It should be noted that although similar diffractionless field propagation has also been reported using graphene solitons enhanced by graphene plasmonics ${ }^{39}$, no nonlinear effect is required in this work as the canalization is due to flatband dispersion enabled by material absorption.

Compared to other integrated diffractionless techniques, the flatband engineering in 2D anisotropic materials can allow field canalization in 2D integrated plasmonic without the need for combining other 3D structures. For instance, propagation of diffraction-free Bessel beam in an integrated, guided-wave setting has been recently reported ${ }^{10}$. However, the generation of such Bessel-type beam based on axicon lens approach requires delicate controls over the phase and amplitude of the propagating waves. As such, phase controlling elements such as optical nanoresonators or photonic crystals should be deployed ${ }^{10,40}$ for the operation of axicon lens. The inclusions of these resonator structures will lead to higher losses, bulkier device footprint, and restricted bandwidth of operation with limited device reconfigurability. Contrarily, our platform based on flatband engineering can represent a more advantageous platform as no such supporting structures are needed in the design. As a whole, anisotropic 2D materials can offer a planar 2D integrated canalization scheme that is readily available in naturally occurring materials, while simultaneously facilitate low loss and subwavelength plasmon propagation with no need to relying on additional 3D structures.

In summary, we have elucidated a physical mechanism where material absorption can be exploited to facilitate dispersion engineering in anisotropic 2D materials. It is found that by regulating material absorption, the dispersion contour can flatten, allowing low-loss plasmon canalization in the subwavelength regime with strong tunability. Some of these capabilities can be achieved in other 2D or 3D metastructures, but not with the dynamic reconfigurability nor performance that a single layer of anisotropic 2D material provides. The realization of such canalization effect can be immediately considered using natural anisotropic 2D materials such as BP. Because the conductivity and the strength of material absorption of BP can be dynamically configured through bias or chemical potential, topological transition process therefore can be observed via bias using BP film. When the dispersion flattens, BP can support low-loss canalization field with a diffraction angle of $5.5^{\circ}$. In addition to near-diffractionless propagation, we also envision that the versatility of dispersion supported in this class of 2D materials, namely capable of supporting elliptic, hyperbolic-like, and mixed type of contour behaviors through bias or doping, can represent a promising material platform to achieve new functionalities for $2 \mathrm{D}$ plasmonic metadevices.

\section{METHODS}

\section{Optical modeling and simulations of 2D plasmonics}

The dispersion plots are obtained by solving Eq. (1) using Mathematica. The simulations of the dipole radiation profile are conducted using Lumerical FDTD, where the electric dipole is placed at the center of the film. The mesh size was set to be $\lambda_{0} / 5000$ around the film due to the deeply subwavelength feature of $2 \mathrm{D}$ materials.

\section{DATA AVAILABILITY}

The data that support the plots and findings within this paper are available from the corresponding author upon reasonable request.

Received: 29 September 2021; Accepted: 1 December 2021; Published online: 13 January 2022

\section{REFERENCES}

1. Maier, S. A. ed. Plasmonics: Fundamentals and Applications (Springer, 2007).

2. Fahrbach, F. O., Gurchenkov, V., Alessandri, K., Nassoy, P. \& Rohrbach, A. Selfreconstructing sectioned bessel beams offer submicron optical sectioning for large fields of view in light-sheet microscopy. Opt. Express 21, 11425-11440 (2013).

3. Dionne, J. A., Diest, K., Sweatlock, L. A. \& Atwater, H. A. Plasmostor: a metal oxide si field effect plasmonic modulator. Nano Lett. 9, 897-902 (2009).

4. Gomez-Diaz, J. S. \& Alu, A. Flatland optics with hyperbolic metasurfaces. ACS Photonics 3, 2211-2224 (2016).

5. Lin, C. C.-C., Chang, P.-H., Su, Y. \& Helmy, A. S. Monolithic plasmonic waveguide architecture for passive and active optical circuits. Nano Lett. 20, 2950-2957 (2020).

6. Boyd, R. ed. Nonlinear Optics 3rd edn (Academic Press, 2008).

7. Durnin, J. Exact solutions for nondiffracting beams: I. the scalar theory. J. Opt. Soc. Am. A 4, 651-654 (1987).

8. Durnin, J., Miceli, J. J. \& Eberly, J. H. Diffraction-free beams. Phys. Rev. Lett. 58, 1499-1501 (1987).

9. Herman, R. M. \& Wiggins, T. A. Production and uses of diffractionless beams. J. Opt. Soc. Am. A 8, 932-942 (1991).

10. Fan, $\mathrm{Y}$. et al. $2 \mathrm{~d}$ waveguided bessel beam generated using integrated metasurface-based plasmonic axicon. ACS Appl. Mater. Interfaces 12, 21114-21119 (2020).

11. Kosaka, H. et al. Self-collimating phenomena in photonic crystals. Appl. Phys. Lett. 74, 1212-1214 (1999).

12. Dellinger, J. et al. Near-field observation of beam steering in a photonic crystal superprism. Opt. Lett. 36, 1074-1076 (2011).

13. Liberal, I. \& Engheta, N. Near-zero refractive index photonics. Nat. Photonics 11, 149-158 (2017).

14. Gomez-Diaz, J. S., Tymchenko, M. \& Alù, A. Hyperbolic plasmons and topological transitions over uniaxial metasurfaces. Phys. Rev. Lett. 114, 233901 (2015).

15. Vakil, A. \& Engheta, N. Transformation optics using graphene. Science 332, 1291-1294 (2011).

16. Li, P. et al. Collective near-field coupling and nonlocal phenomena in infrared phononic metasurfaces for nano-light canalization. Nat. Commun. 11, 3663 (2020).

17. Feng, S. Loss-induced omnidirectional bending to the normal in -near-zero metamaterials. Phys. Rev. Lett. 108, 193904 (2012).

18. Correas-Serrano, D., Alù, A. \& Gomez-Diaz, J. S. Plasmon canalization and tunneling over anisotropic metasurfaces. Phys. Rev. B 96, 075436 (2017).

19. Sun, L., Feng, S. \& Yang, X. Loss enhanced transmission and collimation in anisotropic epsilon-near-zero metamaterials. Appl. Phys. Lett. 101, 241101 (2012). 
20. Shen, N.-H., Zhang, P., Koschny, T. \& Soukoulis, C. M. Metamaterial-based lossy anisotropic epsilon-near-zero medium for energy collimation. Phys. Rev. B 93 245118 (2016).

21. Hu, G., Krasnok, A., Mazor, Y., Qiu, C.-W. \& Alù, A. Moiré hyperbolic metasurfaces. Nano Lett. 20, 3217-3224 (2020).

22. Guangwei, $\mathrm{H}$. et al. Topological polaritons and photonic magic angles in twisted $-\mathrm{MoO}_{3}$ bilayers. Nature 582, 209-213 (2020).

23. Low, T. et al. Tunable optical properties of multilayer black phosphorus thin films. Phys. Rev. B 90, 075434 (2014).

24. Lin, C., Grassi, R., Low, T. \& Helmy, A. S. Multilayer black phosphorus as a versatile mid-infrared electro-optic material. Nano Lett. 16, 1683-1689 (2016).

25. Correas-Serrano, D., Gomez-Diaz, J. S., Melcon, A. A. \& Alù, A. Black phosphorus plasmonics: anisotropic elliptical propagation and nonlocality-induced canalization. J. Opt. 18, 104006 (2016).

26. Li, Y. et al. Plasmonics of $2 \mathrm{~d}$ nanomaterials: properties and applications. Adv. Sci. 4, 1600430 (2017). 1600430-n/a.

27. Engheta, N. Circuits with light at nanoscales: Optical nanocircuits inspired by metamaterials. Science 317, 1698-1702 (2007).

28. Hanson, G. W. Dyadic green's functions for an anisotropic, non-local model of biased graphene. IEEE Trans. Antennas Propag. 56, 747-757 (2008).

29. Mikhailov, S. A. \& Ziegler, K. New electromagnetic mode in graphene. Phys. Rev. Lett. 99, 016803 (2007).

30. Gao, W. et al. Topological photonic phase in chiral hyperbolic metamaterials. Phys. Rev. Lett. 114, 037402 (2015).

31. Coppolaro, M. et al. Extreme-parameter non-hermitian dielectric metamaterials. ACS Photonics 7, 2578-2588 (2020).

32. Lin, C., \& Helmy, A. S. Dynamically reconfigurable nanoscale modulators utilizing coupled hybrid plasmonics. Sci. Rep. 5, 12313 (2015).

33. Su, Y., Chang, P., Lin, C., \& Helmy, A. S. Record Purcell factors in ultracompact hybrid plasmonic ring resonators Sci. Adv. 5, eaav1790 (2019).

34. He, S., Zhang, X. \& He, Y. Graphene nano-ribbon waveguides of record-small mode area and ultra-high effective refractive indices for future VLSI. Opt. Express 21, 30664-30673 (2013).

35. Lin, X. et al. Tailoring the energy distribution and loss of $2 \mathrm{~d}$ plasmons. N. J. Phys. 18, 105007 (2016).

36. Chen, M. et al. Configurable phonon polaritons in twisted a-MoO 3. Nat. Mater. 19 , 1307-1311 (2020).

37. Lin, X. et al. Chiral plasmons with twisted atomic bilayers. Phys. Rev. Lett. 125, 077401 (2020).

38. Zhang, X. et al. Emerging chiral optics from chiral interfaces. Phys. Rev. B 103, 195405 (2021).

39. Nesterov, M. L., Bravo-Abad, J., Nikitin, A. Y., García-Vidal, F. J. \& Martin- Moreno, L. Graphene supports the propagation of subwavelength optical solitons. Laser Photonics Rev. 7, L7-L11 (2013).

40. Zhu, L. et al. Electrically-pumped, broad-area, single-mode photonic crystal lasers. Opt. Express 15, 5966-5975 (2007).

\section{ACKNOWLEDGEMENTS}

This project was funded by the Natural Sciences and Engineering Research Council (NSERC) of Canada.

\section{AUTHOR CONTRIBUTIONS}

P.C. and A.S.H. conceive the idea and perform the analysis showcase in this work. C.L. contributes to BP models that are used in this work. P.C., C.L., and A.S.H. analyzed and wrote the manuscript.

\section{COMPETING INTERESTS}

The authors declare no competing interests.

\section{ADDITIONAL INFORMATION}

Supplementary information The online version contains supplementary materia available at https://doi.org/10.1038/s41699-021-00283-4.

Correspondence and requests for materials should be addressed to Po-Han Chang or Amr S. Helmy.

Reprints and permission information is available at http://www.nature.com/ reprints

Publisher's note Springer Nature remains neutral with regard to jurisdictional claims in published maps and institutional affiliations.

Open Access This article is licensed under a Creative Commons Attribution 4.0 International License, which permits use, sharing, adaptation, distribution and reproduction in any medium or format, as long as you give appropriate credit to the original author(s) and the source, provide a link to the Creative Commons license, and indicate if changes were made. The images or other third party material in this article are included in the article's Creative Commons license, unless indicated otherwise in a credit line to the material. If material is not included in the article's Creative Commons license and your intended use is not permitted by statutory regulation or exceeds the permitted use, you will need to obtain permission directly from the copyright holder. To view a copy of this license, visit http://creativecommons. org/licenses/by/4.0/.

C The Author(s) 2022 